\title{
PITFALLS IN CALCULATING SCATTERING BY SMALL PARTICLES
}

\author{
DONALD R. HUFFMAN \\ Department of Physics, University of Arizona
}

\begin{abstract}
Comparisons between measured extinction on small particle systems and the same quantity derived from scattering calculations using bulk optical constants often show substantial disagreements. Based on such comparisons, certain pitfalls which have been recognized in such a process, as it applies to the modelling of interstellar dust properties, are pointed out. Illustrations are taken from laboratory work on $S i C$, graphitic carbon, and olivine-type silicates.
\end{abstract}

\section{INTRODUCTION}

Models of optical effects due to interstellar dust usually invoke Mie scattering theory using laboratory measurements of optical constants. Other treatments of scattering in the case of nonspherical particles or clusters of particles are also used. The everpresent question is, "How good is such an approach in modelling the true situation observed in nature?" For many years we have pursued a program of experimental work in which we measure bulk optical constants for a particular solid (if they are not available), use these to calculate small particle properties such as scattering and absorption, and then produce small particles of the material and measure the same optical properties. It is seldom that the two ways of arriving at the same optical properties agree very well. In the process of this experimental work we have come to recognize certain problems that frequently occur in the process. These pitfalls are the subject of this paper.

\section{A DISCUSSION OF SOME PITFALLS}

Optical constants required as input to scattering calculations usually come from laboratory measurements, which are found dispersed through a broad collection of scientific journals and handbooks. The difficulties and uncertainties involved in measuring optical constants are seldom appreciated by the mere user of the data. An excellent compilation of optical constants which also contains critical reviews of methods and uncertainties involved in optical constants measurements is contained in the volume edited by Palik (1985). Although it will probably not contain any new solids of interest to astronomers, it is recommended reading. Because of the difficulties involved in measuring optical constants, the first pitfall that must be recognized is that they may simply be wrong. 
Another pitfall regarding optical constants is that they often depend strongly on the degree of disorder present in the material, so that available measurements made on a single crystal solid may be a poor representation of a small particle of the same chemical composition. In some fortunate cases, optical constants may have been measured for both single crystal and for amorphous forms of the solid, but even here the interstellar particle may be intermediate in degree of disorder. Carbon and the silicate olivine, to be presented in the sections that follow, are perhaps the best examples of this difficulty.

It is fairly obvious when one considers particles of smaller and smaller size that there will be some small size at which the optical constants of bulk material cease to be valid. The optical constants of bulk carbon of any form can never describe the molecular spectrum of polyatomic carbon. This can be impressively illustrated by comparing the rich and complicated absorption spectrum shown by graphite vapor trapped as molecular clusters (Krätschmer, Sorg, and Huffman, 1984) with the smooth spectrum of graphite particulate extinction (Figure 2 of this paper for example). I have dealt with the problem of the inevitable breakdown of bulk optical constants (Huffman, 1988) and have concluded that, below about $50 \AA$ in particle size one should start worrying about whether the bulk optical constants provide an adequate representation of small particle behavior.

Because of difficulties such as these in using bulk optical constants and Mie calculations there is a tendency to want to simply use direct optical measurements on the small particles of interest - measurements such as spectral dependence of extinction or emission. Although this at first sounds like an ideal solution, there are severe difficulties in creating the same conditions in the laboratory that one might find in interstellar space. The two dominant problems we have run into in this approach are interaction of the particles with the substrate and particle clustering or aggregation. Both of these can give much different results from what is expected for isolated particles.

\section{AN EXAMPLE FROM THE LABORATORY}

The results of one of our attempts to achieve agreement between measured extinction and the same quantity calculated from optical constants is shown in Figure 1 (from Bohren and Huffman, 1983). The solid of interest is $S i C$ for which optical constants for the common hexagonal form of the solid were measured by Spitzer et al. (1959). In order to compare with sphere calculations shown in the figure, measurements were made on an experimental sample of sub-micron sized particles selected by gravitational settling and dispersed thoroughly in a transparent $\mathrm{KBr}$ matrix. When plotted on the same volume-normalized extinction scale, there is extremely poor agreement between measurements and sphere calculations. As was first pointed out by D. P. Gilra (in calculations included in Treffers and Cohen, 1974), nonspherical shape has a very large effect on the extinction for solids like $S i C$ in regions where the real part of the dielectric function goes strongly negative. Instead of a single sphere mode dominating as shows up in the sphere calculations, the irregular particles give rise to many different shape factors, which produce a spread of modes primarily in the region where the real part of the dielectric function goes negative. In order to roughly represent the effect of this distribution of shape factors, Bohren and I developed what we call the continuous distribution of ellipsoid shapes (CDE) calculation, which assumes that all possible shape factors 


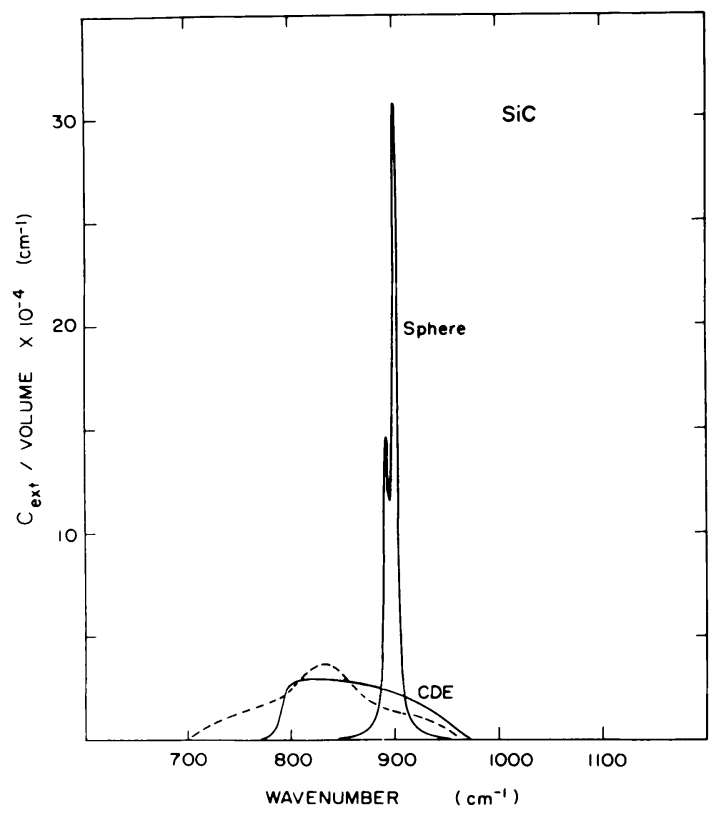

Fig. 1. Measured extinction by $\mathrm{SiC}$ particles (dashed curve) compared with calculations (from Bohren and Huffman, 1983).

of an ellipsoid are present in a highly irregular sample. The result is a simple one line expression for calculating volume-normalized extinction for irregular particles in the Rayleigh size regime. Details and other examples are found in chapter 12 of our monograph (Bohren and Huffman, 1983). Although, admittedly a great oversimplification, we have been pleasantly surprised at how much better it seems to fit the experimental facts than does sphere theory in these difficult spectral regions. The improved agreement seems to apply for a distribution of shape factors caused either by highly irregular particle shapes or by clustering of spherical particles into irregular agglomerates.

It should not be inferred that the discrepant results of calculations in Figure 1 are representative of all solids in all wavelength ranges. For most common solids in the visible there is very little difference between the CDE calculations and sphere calculations, because the optical constants are rather "normal". One of the valuable uses of the CDE approach seems to be that one can quickly estimate the probable effect of nonsphericity and/or clustering for particles in the Rayleigh size regime by comparing the CDE and sphere calculations. I will use this sort of comparison for the two examples that follow. 


\section{GRAPHITE AND THE $2200 \AA$ BAND}

As best I can tell from looking at the early literature on the subject and from talking with Ted Stecher during the course of this meeting, the graphite hypothesis for explanation of the $2200 \AA$ bump (Stecher and Donn, 1965) followed close on the heels of the discovery of the feature (Stecher, 1965). Amazingly, the first reported observations showed the same essential position and shape of the bump that we know now. In addition to the insight of Stecher and Donn, the hypothesis was greatly aided by the fortuitous circumstance of the publication of graphite optical constants into the far ultraviolet by Taft and Philipp (1965). Taft and Philipp were established solid state physicists, operating with a new generation of far UV optical instruments. Industry had provided ideal samples in the form of stressannealed pyrolytic graphite, which could be easily cleaved (using cellophane tape) to produce atomically clean and smooth surfaces. Thus Stecher and Donn were able to publish their Mie calculations on graphite, which happily produced a rather good fit to the $2200 \AA$ bump.

With this background I now refer to Figure 2 which gives the mass normalized extinction data measured by Otto Edoh (1983) for graphitic carbon smoke condensed from vaporized graphite, along with several calculations. The measurements are a more recent set similar to those published earlier (Day and Huffman, 1973; Huffman, 1977; Stephens, 1980), with the innportant improvement that they have been carefully mass-normalized to preclude the need for an arbitrary scaling parameter. Using the two sets of optical constants for single crystal graphite, Edoh's calculations were made for spheres and for the distribution of ellipsoidal shapes, both in the Rayleigh limit. It can be seen that there is a considerable shift and broadening of the band as more complicated shape factors are included. This is not surprising in view of the results in Figure 1. Calculations in Figure 2 also are shown using the glassy carbon optical constants (Arakawa et al., 1977). This represents a more disordered but not amorphous form of carbon. Note that the two calculations for glassy carbon are very similar. Comparing the measurements with these calculations one can see that improved agreement results from invoking either a distribution of shapes or clustering (by means of the CDE theory) in graphite, or by using the less crystalline glassy carbon properties. The surprising additional information that one gets, however, is that the measured mass-normalized extinction is much stronger than can be explained by glassy carbon. In fact it is even stronger than what is predicted for single crystal graphite.

Several conclusions are possible from this comparison. First, I can easily understand why our laboratory extinction measurements do not give the position and the width which is correct to match either graphite Mie calculations or the interstellar extinction curve. The difficulties are probably the same as those involved in the work of Figure 1 - clustering of particles on a substrate. Until we are able to do the difficult job of measuring extinction on a cloud of the smoke particles which has not been allowed to agglomerate, we probably can't expect much better. However, invoking more disordered carbon does not seem to help. In fact, the particles we make in the laboratory, which have often been speculated to be much more highly disordered than graphite, seem to have about the same optical absorption strength in this region as single crystal graphite.

Because of this, I conclude that a graphitic carbon not too different from graphite 


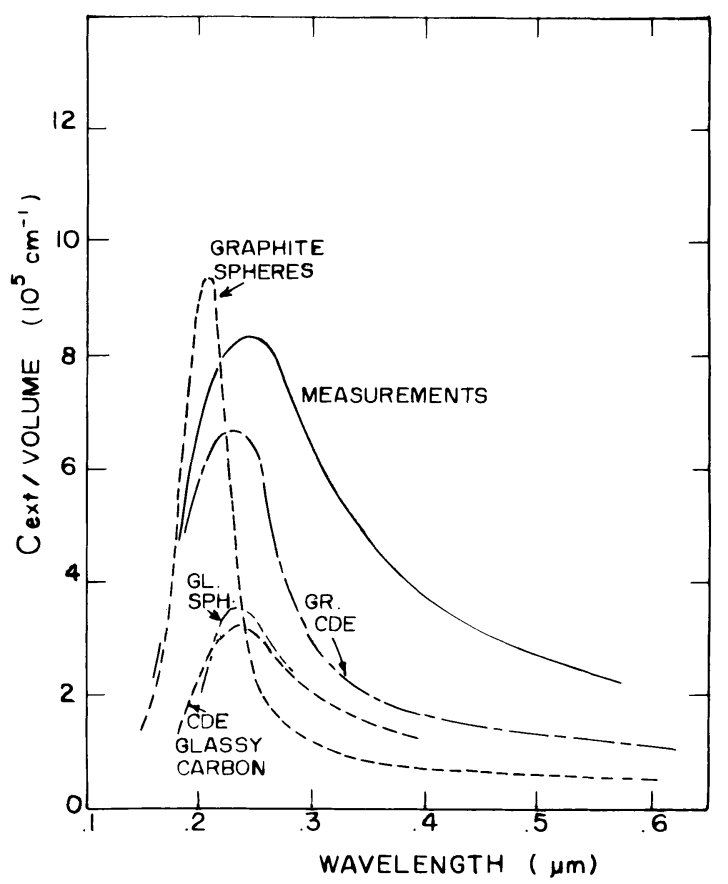

Fig. 2. Calculations of extinction for graphite (gr) and for glassy carbon particles (gl) compared with measurements on graphitic carbon smoke (from Edoh, 1983).

is still the most reasonable candidate to explain the $2200 \AA$ band, as Stecher and Donn suggested a long time ago.

\section{OLIVINE SILICATES AND THE $10 \mu \mathrm{m}$ FEATURE}

The first tries at using optical constants to model the $10 \mu \mathrm{m}$ interstellar and circumstellar feature did not work out as well as the early successes of the graphite calculations. At the time the $10 \mu \mathrm{m}$ band was first observed, there were no good optical constants available for the type of silicates that were predicted by condensation calculations. The predictions favored condensates of the olivine family with chemical composition of the form $(\mathrm{Mg}, \mathrm{Fe})_{2} \mathrm{SiO}_{4}$ (for example Grossman, 1972). Terry Steyer in my lab did the large amount of work necessary to determine the three sets of optical constants for high quality, single crystal olivine. This set of optical constants is one of the most complete ever measured for such a triaxial crystal. Unfortunately, the first round of Mie calculations using these optical constants showed sharp structure that would obviously never explain the interstellar band. Sphere calculations using Steyer's optical constants are shown in Figure 3 along with CDE calculations. Because of the sharp structure apparent in the sphere calculations, the work that had taken Steyer nearly three years to complete was thrown aside in a very short time. 


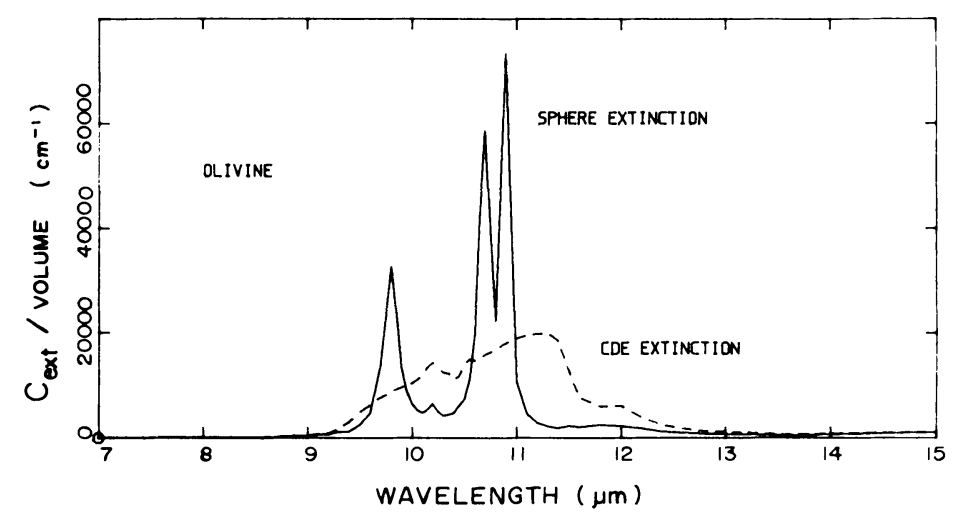

Fig. 8. Extinction calculated for particles of crystalline olivine using optical constants from Steyer (1974).

The obvious direction to look was toward more amorphous silicates which would have less sharp structure. Krätschmer and I (1979) heavily irradiated the single crystals of olivine that Steyer had used and determined optical constants of the resulting damaged layer. In addition, Day (1979) produced amorphous silicates (including $\mathrm{Mg}_{2} \mathrm{SiO}_{4}$ ) in thin films and determined optical constants. In both cases the sharp structure of crystalline olivine had vanished, making more favorable the comparison between emission and absorption calculated from optical constants and the astronomical observations. It also proved possible to produce and measure directly the extinction for small, disordered olivine particles. When one vaporizes the single crystal olivine that Steyer used in his optical constants measurements and allows it to recondense as smoke in air, the extinction frequently shows a structureless band similar to what one calculates. Such measurements on the disordered olivine smoke are shown in Figure 4 along with several calculations. The experimental spectrum agrees more favorably with calculations based on Day's optical constants than with calculations (not included) based on the irradiated olivine optical constants. This probably indicates that the former represents more highly disordered olivine than the latter.

Calculations based on Day's optical constants are shown in Figure 4 for both spheres and for the distribution of ellipsoids, both in the Rayleigh limit. Note that, in comparison to the crystalline calculations of Figure 3, there is comparatively little difference between the sphere and CDE calculations for disordered olivine. Based on our understanding of experiments such as the one summarized in Figure 1, these results suggest that neither shape effects for particles in space nor clustering for samples in the laboratory are expected to produce much of a problem. Pitfalls are sometimes present and sometimes not.

The optical constants for "astronomical silicate" (Draine and Lee, 1984) show an even broader feature which may mean that the degree of disorder is even greater than has been achieved in any of the olivine-type laboratory silicates, as one can see by the included calculations in Figure 4.

During the past couple of years interest has revived in the properties of crys- 


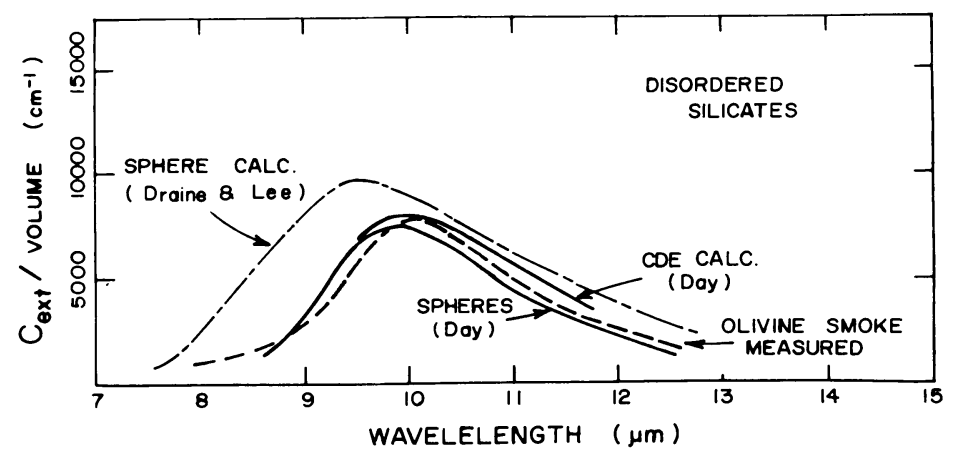

Fig. 1. Calculated extinction for disordered silicates using different sets of optical constants. Experimental extinction for disordered olivine smoke is also included.

talline silicate because of the infrared observations from Halley's comet (Bregman et al., 1987), which seem to show several bands in the infrared attributable to olivine. In addition to a crystalline olivine-like feature near $11.2 \mu \mathrm{m}$, another component seems to be necessary to match a broad hump peaking near $9.7 \mu \mathrm{m}$. The results of our Figures 3 and 4 suggest some interesting insights into this problem and the possible connection between Halley dust and interstellar dust. First, the sphere calculations for crystalline olivine indeed show structure in the $11 \mu \mathrm{m}$ region, but the peaks are well shortward of $11 \mu \mathrm{m}$, whereas the Halley peak is at about 11.2 $\mu \mathrm{m}$. If the CDE calculation is more appropriate to aggregated particles in Halley, this could explain why the peak in the calculated CDE extinction is nearly coincident with the observed comet feature. In fact, the infrared spectra of several interplanetary dust particles, analyzed to be predominantly olivine (Sandford and Walker, 1985), look surprisingly similar to our CDE calculations of Figure 2 and very dissimilar to the sphere calculations. Since these IDP's are aggregates of very small olivine grains compacted in the sample preparation process, the comparison to the CDE calculations may be analogous to the comparisons in Figure 1. The additional broad $9.7 \mu \mathrm{m}$ feature of Halley could be due to a highly amorphous component of the same type of olivine silicate that forms the sharper feature. Under this hypothesis, a pretty good match to the Halley dust data comes from conjuring up highly amorphous silicate particles, (as in Figure 4) such as appear to populate various regions of the interstellar medium, letting them agglomerate into a loose aggregate, and annealing a portion of it into a crystalline form of the same silicate (as in the CDE calculations of Figure 3).

Whether right or wrong, it is hoped that this playing around with measured silicate optical constants has illustrated some of the potential pitfalls we have tried to present in this paper - in particular the problem of varying optical constants with crystallinity and the potential problems of clustering and/or nonsphericity.

\section{REFERENCES}

Arakawa, E. T., Williams, M. W., and Inagaki, T. 1977, J. Appl. Phys., 48, 3176. 
Bregman, J. D., Campins, H., Witteborn, F. C., Wooden, D. H., Rank, D. M., Allamandola, L. J., Cohen, M., and Tielens, A. G. G. M. 1987, Astr. Ap., 187, 616.

Bohren, C. F., and Huffman, D. R. 1983, Absorption and Scattering of Light by Small Particles, (New York: Wiley).

Day, K. L., and Huffman, D. R. 1973, Nature Phys. Sci., 243, 50.

Day, K. L. 1979, Ap. J., 284, 158.

Draine, B. T., and Lee, H. M. 1984, Ap. J., 285, 89.

Edoh, O. 1983, Ph.D. Thesis, University of Arisona.

Grossman, L. 1972, Geochim. Cosmochim. Acta., 86, 597.

Huffman, D. R. 1977, Adv. Phys., 28, 129.

Huffman, D. R. 1988, in Optical Effects Associated with Small Particles, eds. P. W. Barker and R. K. Chang, (Singapore: World Scientific), p. 279.

Krätschmer, W., and Huffman, D. R. 1979, Ap. Space Sci., 61, 195.

Krätschmer, W., Sorg, N., and Huffman, D. R. 1985, Surface Sci., 156, 814.

Palik, E. D. (ed.) 1985, Handbook of Optical Constants of Solids, (New York: Academic).

Sandford, S. A., and Walker, R. M. 1985, Ap. J., 291, 838.

Spitser, W. G., Kleinman, D., and Walsh, D. 1959, Phys. Rev., 113, 127.

Stecher, T. 1965, Ap. J., 142, 1683.

Stecher, T., and Donn, B. 1965, Ap. J., 142, 1682.

Stephens, J. R. 1980, Ap. J., 287, 450.

Steyer, T. R. 1974, Ph.D. Thesis, University of Arizona.

Taft, E. A., and Philipp, H. R. 1965, Phys. Rev., 188, A197.

Treffers, R., and Cohen, M. 1974, Ap. J., 188, 545. 